\title{
Contraction and Expansion of Convex Sets
}

\author{
Michael Langberg • Leonard J. Schulman
}

Received: 5 October 2007 / Revised: 15 April 2009 / Accepted: 17 July 2009 /

Published online: 4 August 2009

(C) Springer Science+Business Media, LLC 2009

\begin{abstract}
Let $\mathcal{S}$ be a set system of convex sets in $\mathbb{R}^{d}$. Helly's theorem states that if all sets in $\mathcal{S}$ have empty intersection, then there is a subset $\mathcal{S}^{\prime} \subset \mathcal{S}$ of size $d+1$ which also has empty intersection. The conclusion fails, of course, if the sets in $\mathcal{S}$ are not convex or if $\mathcal{S}$ does not have empty intersection. Nevertheless, in this work we present Helly-type theorems relevant to these cases with the aid of a new pair of operations, affine-invariant contraction, and expansion of convex sets.

These operations generalize the simple scaling of centrally symmetric sets. The operations are continuous, i.e., for small $\varepsilon>0$, the contraction $C^{-\varepsilon}$ and the expansion $C^{\varepsilon}$ are close (in the Hausdorff distance) to $C$. We obtain two results. The first extends Helly's theorem to the case of set systems with nonempty intersection:

(a) If $\mathcal{S}$ is any family of convex sets in $\mathbb{R}^{d}$, then there is a finite subfamily $\mathcal{S}^{\prime} \subseteq \mathcal{S}$ whose cardinality depends only on $\varepsilon$ and $d$, such that $\bigcap_{C \in \mathcal{S}^{\prime}} C^{-\varepsilon} \subseteq \bigcap_{C \in \mathcal{S}} C$.

The second result allows the sets in $\mathcal{S}$ a limited type of nonconvexity:

(b) If $\mathcal{S}$ is a family of sets in $\mathbb{R}^{d}$, each of which is the union of $k$ fat convex sets, then there is a finite subfamily $\mathcal{S}^{\prime} \subseteq \mathcal{S}$ whose cardinality depends only on $\varepsilon, d$, and $k$, such that $\bigcap_{C \in \mathcal{S}^{\prime}} C^{-\varepsilon} \subseteq \bigcap_{C \in \mathcal{S}} C$.
\end{abstract}

Keywords Helly-type theorems · Nonconvex

Research supported in part by grants from the NSF and the NSA.

M. Langberg $(\bowtie)$

Computer Science Division, The Open University of Israel, Raanana 43107, Israel

e-mail:mikel@openu.ac.il

\section{L.J. Schulman}

Department of Computer Science, California Institute of Technology, Pasadena, CA 91125, USA

e-mail: schulman@caltech.edu 


\section{Introduction}

Helly's theorem is one of the fundamental results in discrete geometry [12]. It states that if every $\leqslant d+1$ sets in a set system $\mathcal{S}$ of compact convex sets in $\mathbb{R}^{d}$ have nonempty intersection, then all of the sets in $\mathcal{S}$ have nonempty intersection. Equivalently, if the entire family $\mathcal{S}$ has empty intersection, then there is a subset $\mathcal{S}^{\prime} \subset \mathcal{S}$ (a witness) of size $\leqslant d+1$ which also has empty intersection. Over the years the basic Helly theorem has spawned numerous generalizations and variants (e.g., [7, 21]). These have the following local-global format: If every $m$ members of a family have property $P$, then the entire family has property $P$ (or sometimes a weaker property $P^{\prime}$ ). Equivalently, if the entire family has property $P^{\prime c}$, then there is a witness subfamily of size $m$ having the (possibly weaker) property $P^{c}$.

The conclusion of Helly's theorem fails, of course, if the sets in $\mathcal{S}$ are not convex; also if one changes the property "empty intersection" to notions of "small intersection." Nevertheless, we present Helly-type theorems that apply to cases of these sorts. We do so by allowing in the local-global transition not a weakening of the property $P$ but arbitrarily slight changes in the sets themselves. We use a pair of operations, the contraction $C^{-\varepsilon}$, and expansion $C^{\varepsilon}$ of a convex set $C$. For centrally symmetric convex sets, these are simply homothetic scalings with respect to the center by factor $(1+\varepsilon)$ and $(1-\varepsilon)$, respectively; for general convex sets, the definitions are very natural and seem not to have been used before. Our operations and Helly-type theorems are described below.

\subsection{Expansion and Contraction of Convex Sets}

Throughout the main body of the paper all sets are subsets of $\mathbb{R}^{d}$, and the convex sets we consider are compact (i.e., closed and bounded). Unbounded sets and sets that are not necessarily closed will be discussed in Sect. 6. For two points $x$ and $y$ in $\mathbb{R}^{d}$, the line segment between $x$ and $y$ is denoted by $x y$, and its length by $|x y|$. Let $S^{d-1}$ be the unit sphere in $\mathbb{R}^{d}$. For a set $A \subseteq \mathbb{R}^{d}$ and $c \in \mathbb{R}$, the set $c A$ is $\{c x \mid x \in A\}$. Given two sets $A$ and $B$, define the binary operations "+" and "-" (Minkowski sum and difference) by $A+B=\{x+y \mid x \in A, y \in B\}$ and $A-B=A+(-B)$. For a unit vector $u$, a $u$-hyperplane is a $(d-1)$-dimensional affine subspace perpendicular to $u$. A slab is the Minkowski sum of a hyperplane and a finite segment, and a $u$-slab is one bounded by $u$-hyperplanes. The $u$-slab of a set $A$ is the closed $u$-slab of minimal width containing $A$; it is denoted $s_{u}(A)$.

Let us recall the standard definition of contraction and expansion for centrally symmetric sets. A convex set $C \subseteq \mathbb{R}^{d}$ is centrally symmetric if it has a center $p$ such that $C=2 p-C$. For a centrally symmetric convex set $C$, let $\|x\|_{C}$ be the norm of $x$ with respect to $C:\|x\|_{C}=\inf \left\{r>0 \mid \frac{x-p}{r}+p \in C\right\}$. Now, for any $\varepsilon \geqslant-1$, define $C^{\varepsilon}=\left\{x \mid\|x\|_{C} \leqslant 1+\varepsilon\right\}$. Namely, for positive $\varepsilon$, the set $C^{\varepsilon}$ is a blown-up version of $C$ and is referred to as the expansion of $C$; and for negative $\varepsilon$, the set $C^{\varepsilon}$ is a shrunken version of $C$ and is referred to as the contraction of $C$ (see Fig. 1). As $\varepsilon$ tends to 0 , the set $C^{\varepsilon}$ tends to $C$. It is not hard to verify that $C^{\varepsilon}$ is convex. Notice the distinction between $C^{\varepsilon}$ and the Minkowski sum $C+\varepsilon \mathcal{B}$, where $\mathcal{B}$ is the unit ball centered at the origin; the first definition commutes with affine transformations, the second does not. 


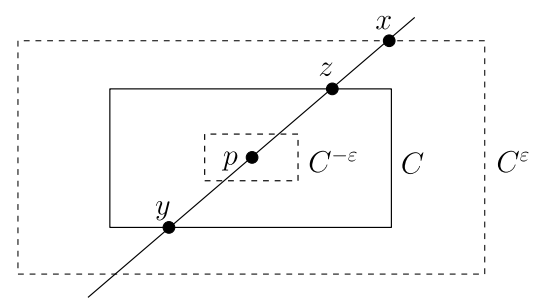

Fig. 1 An illustration of the contraction and expansion of a centrally symmetric convex set $C$. The set $C$ with center $p$ is given by a solid line. For positive $\varepsilon$, the expansion $C^{\varepsilon}$ and the contraction $C^{-\varepsilon}$ of $C$ are given by dashed lines. The point $x$ is outside $C$. The points $y$ and $z$ are on the boundary of $C$ and the line passing through $x$ and $p$. It can be seen that $\|x\|_{C}=\frac{|p x|}{|p z|}$

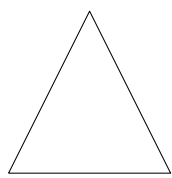

$C$

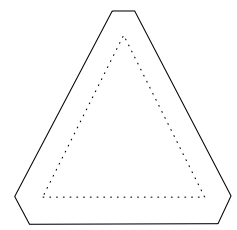

$C^{\varepsilon}$

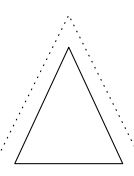

$C^{-\varepsilon}$

Fig. 2 An illustration of Definition 1.1 applied to a triangle $C$ (which is not centrally symmetric). Here $\varepsilon>0$. In the presentation of $C^{\varepsilon}$ and $C^{-\varepsilon}$, the set $C$ is drawn with a dotted line, and its expansion/contraction is drawn with a solid line. Notice that the expansion $C^{\varepsilon}$ of $C$ is no longer a triangle

We are now ready to define contraction and expansion of general compact convex sets $C$. First notice that we cannot use a direct analog to the definition for centrally symmetric bodies as a general convex set lacks a center point $p$. We thus consider an alternative definition to $C^{\varepsilon}$ for centrally symmetric $C$ which is independent of $p$, and then extend it to general convex sets. Let $C$ be centrally symmetric around the origin. Let $u$ be any unit vector in $\mathbb{R}^{d}$. Consider the $u$-slab $s_{u}(C)$ of $C$. Clearly, $C \subseteq s_{u}(C)$. Moreover, $C=\bigcap_{u \in S^{d-1}} s_{u}(C)$. Finally, it is not hard to verify that $C^{\varepsilon}$ as defined above is equal to $\bigcap_{u} s_{u}^{\varepsilon}(C)$. Thus we conclude an alternative equivalent definition for contraction and expansion of centrally symmetric convex sets: $C^{\varepsilon}=\bigcap_{u} s_{u}^{\varepsilon}(C)$. Namely, a definition which relies solely on the notion of contraction and expansion of slabs. This is the definition we shall use for general convex sets.

Definition 1.1 (Contraction and Expansion) Let $C$ be a compact convex set, and let $\varepsilon \geqslant-1$ be any real (positive, negative, or zero). Let $C^{\varepsilon}=\bigcap_{u \in S^{d-1}} s_{u}^{\varepsilon}(C)$. For any $x \in \mathbb{R}^{d}$, let $\|x\|_{C}$ be $1+\varepsilon$ for the minimum $\varepsilon$ such that $x \in C^{\varepsilon}$. We stress (as opposed to the case that $C$ is centrally symmetric about the origin) that $\|\cdot\|_{C}$ is not necessarily a norm. The definition above is illustrated in Fig. 2.

We are now ready to present our Helly-type theorems that rely on the definitions above. The theorems have the following structure: if a given set family $\mathcal{S}$ has property $P$, then there is a witness subfamily $\mathcal{S}^{\prime}$ of size $s$ whose contraction also has property $P$. 


\subsection{Finding a Witness for Small Intersection}

Consider the case in which the given set system $\mathcal{S}$ consists of convex sets, however their intersection is not empty. In this case, as an analog to Helly's theorem, one may seek a witness of small cardinality $\mathcal{S}^{\prime} \subseteq \mathcal{S}$ whose intersection is contained in the intersection of the sets of $\mathcal{S}$. It is not hard to verify that finite witnesses do not exist even for systems of convex sets in $\mathbb{R}^{2}$. For example, for every unit vector $u \in \mathbb{R}^{2}$, let $C_{u}$ be the strip of width 2 consisting of vectors $v$ with $\langle v, u\rangle \in[-1,1]$. The intersection of the family $\mathcal{S}=\left\{C_{u}\right\}$ is the closed unit ball $\mathcal{B}$ centered at the origin, and any finite subset $\mathcal{S}^{\prime}$ of this family has intersection which strictly includes $\mathcal{B}$. Namely, no finite witness for the intersection of $\mathcal{S}$ exists. We show:

Theorem 1.1 Let $\varepsilon>0$. Let $\mathcal{S}=\left\{C_{i}\right\}$ where each $C_{i}$ is compact and convex in $\mathbb{R}^{d}$. There exists a subset $\mathcal{S}^{\prime}$ of $\mathcal{S}$ of size at most $s(d, \varepsilon)=\frac{(c d)^{d}}{\varepsilon^{\frac{d}{2}}}$ such that $\bigcap_{C \in \mathcal{S}^{\prime}} C^{-\varepsilon} \subseteq$ $\bigcap_{C \in \mathcal{S}} C$. Here $c>0$ is a universal constant.

We note that $s(d, \varepsilon)$ in Theorem 1.1 is close to tight as a function of $\varepsilon$ (see Sect. 4).

\subsection{Sets that are not Necessarily Convex}

Now, consider the case in which the set system $\mathcal{S}$ does not consist of convex sets, but rather of sets that are the union of a bounded number of convex sets. Does the natural analog of Helly's theorem hold for such systems $\mathcal{S}$ ? Namely, if $\bigcap_{C \in \mathcal{S}} C$ is empty, is there a small witness $\mathcal{S}^{\prime} \subseteq \mathcal{S}$ for this fact? As before, it is not hard to verify that the answer is no-even in the simplest case where all sets in $\mathcal{S}$ consist of the union of two convex sets in $\mathbb{R}$. For example, consider the family $\mathcal{S}=\left\{C_{1}, \ldots, C_{n-1}\right\}$ in which $C_{i}$ is the closure of $[0,1] \backslash\left[\frac{i-1}{n}, \frac{i+1}{n}\right]$. Set difference is denoted " $\backslash$ ". Each $C_{i}$ is the union of at most two closed intervals, and $\bigcap C_{i}=\emptyset$. However, any strict subfamily $\mathcal{S}^{\prime}$ of $\mathcal{S}$ has a nonempty intersection.

For real $f \geqslant 1$, a bounded convex set $C$ is $f$-fat if the ratio between the radii of the minimum radius ball containing $C$ and the maximum radius ball contained in $C$ is at most $f$ (see, e.g., [8]; this is essentially inverse to other definitions, e.g., [20]). For a set $C$ consisting of the union of $k$ convex sets $\left\{C_{1}, \ldots, C_{k}\right\}$, define $C^{-\varepsilon}$ to be $\bigcup_{i=1}^{k} C_{i}^{-\varepsilon}$. Observe that the definition depends on the constituents $C_{i}$ and not only on their union. We show:

Theorem 1.2 Let $\varepsilon>0$. Let $\mathcal{S}=\left\{C_{i}\right\}$ where each $C_{i}$ is the union of at most $k f$-fat compact convex sets in $\mathbb{R}^{d}$. There exists a subset $\mathcal{S}^{\prime}$ of $\mathcal{S}$ of size at most $s(k, d, \varepsilon, f)=$ $k !\left(\frac{c^{k} d f^{k-1}}{\varepsilon^{k-\frac{1}{2}}}\right)^{d}$ such that $\bigcap_{C \in \mathcal{S}^{\prime}} C^{-\varepsilon} \subseteq \bigcap_{C \in \mathcal{S}}$ C. Here $c>0$ is a universal constant.

A few remarks are in order. First notice that if $\bigcap_{C \in \mathcal{S}} C=\emptyset$, then Theorem 1.2 states the existence of a small witness $\mathcal{S}^{\prime}$ for empty intersection (extending Helly's theorem). Secondly, in $\mathbb{R}$, all bounded convex sets are 1 -fat, so the fatness condition is not a restriction in $d=1$. We conjecture that the fatness condition is unnecessary also in higher dimension (Conjecture 1.1). Finally, in $\mathbb{R}$ we are able to improve the 
value $s(k, 1, \varepsilon, 1)$ to approximately $(c / \varepsilon)^{k / 2} \log ^{k / 2}(1 / \varepsilon)$ for some constant $c>0$. This value of $s(k, 1, \varepsilon, 1)$ can be shown to be nearly tight as a function of $\varepsilon$ (see Sects. 3 and 4).

Conjecture 1.1 Let $\mathcal{S}=\left\{C_{i}\right\}$ where each $C_{i}$ is the union of at most $k$ compact convex sets in $\mathbb{R}^{d}$. There exists a subset $\mathcal{S}^{\prime}$ of $\mathcal{S}$ whose size depends only on $k, d$, and $\varepsilon$ such that $\bigcap_{C \in \mathcal{S}^{\prime}} C^{-\varepsilon} \subseteq \bigcap_{C \in \mathcal{S}} C$.

\subsection{Related Work}

To the best of our knowledge, these contraction and expansion operations for convex sets (except in the centrally symmetric case) have not previously been considered. Also, we are not aware of other Helly-type theorems which apply to the case of nonempty intersection.

We note that Minkowski difference with a unit ball differs substantially from our definition of contraction and cannot be used as an alternative notion of contraction in Theorems 1.1 and 1.2. Namely, our definition commutes with affine transformations, while the Minkowski difference with $\varepsilon \mathcal{B}$ does not. More specifically, notice that for "large" convex sets $C$, the set $C-\varepsilon \mathcal{B}$ may be very "close" to $C$, while for "small" $C, C-\varepsilon \mathcal{B}$ may differ significantly from $C(C-\varepsilon \mathcal{B}$ may even be empty). Thus using $C-\varepsilon \mathcal{B}$ instead of $C^{-\varepsilon}$ in our theorems will not allow one to define a witness size $s(\varepsilon, d)$ which is independent of the family $\mathcal{S}$ at hand. Our notion of contraction overcomes this difficulty.

There is an interesting literature on Helly-type theorems for unions of convex sets. (For a nice survey on Helly-type theorems in general, see Eckhoff [7] or Wenger [21].) Let $\mathcal{C}_{k}^{d}$ be the family of all sets in $\mathbb{R}^{d}$ that are the union of at most $k$ convex sets. The intersection of members in $\mathcal{C}_{k}^{d}$ are not necessarily in $\mathcal{C}_{k}^{d}$, and in general, as we have noted, subfamilies of $\mathcal{C}_{k}^{d}$ do not have finite Helly number (i.e., there is not a finite witness for empty intersection). Nevertheless, it was shown independently by Matoušek [16] and Alon and Kalai [1] that if $\mathcal{S}$ is a finite subfamily of $\mathcal{C}_{k}^{d}$ such that the intersection of every subfamily of $\mathcal{S}$ is in $\mathcal{C}_{k}^{d}$, then $\mathcal{S}$ has finite Helly number. Let $\mathcal{K}_{k}^{d}$ be the family of all sets in $\mathbb{R}^{d}$ that are the union of at most $k$ pairwise disjoint convex sets. As before, $\mathcal{K}_{k}^{d}$ does not have finite Helly number. Helly-type theorems for subfamilies $\mathcal{S}$ of $\mathcal{K}_{k}^{d}$ such that the intersection of every subfamily of $\mathcal{S}$ is in $\mathcal{K}_{k}^{d}$ have been studied. Grunbaum and Motzkin [11] showed that for $k=2$, the Helly number of such $\mathcal{S}$ is $2(d+1)$, and for general $k$, conjectured it to be $k(d+1)$ (which is tight). The case $k=3$ was proven by Larman [15], and the general case by Morris [17]. An elegant proof (based on the notion of LP-type problems) was presented by Amenta [2] and recently generalized to a topological setting in [14] . Differently from this literature, our results do not depend on a restriction on the intersections of subfamilies of $\mathcal{S}$.

Let $\mathcal{S}=\left\{C_{i}\right\}$ be a family of convex sets in $\mathbb{R}^{d}$. In [3], it is shown for a certain function $v(d) \simeq d^{-d / 2}$, that if the intersection of any $2 d$ or fewer members of $\mathcal{S}$ has volume at least 1 , then the volume of $\bigcap_{\mathcal{S}} C_{i}$ is at least $v(d)$. This statement has a similar quantitative flavor to the following immediate consequence of Theorem 1.1: If for every $\mathcal{S}^{\prime}$ of size $s(d, \varepsilon)$, the intersection $\bigcap_{C \in \mathcal{S}^{\prime}} C^{-\varepsilon}$ has volume at least $v$, then 
$\bigcap_{C \in \mathcal{S}} C$ has volume at least $v$. Despite this similarity, the results of this work (and our proof techniques) differ substantially from those of [3].

Finally, in a recent and independent paper [5], the problem of approximately covering a given convex set $U$ by a small subset $\mathcal{F}^{\prime}$ of a convex set system $\mathcal{F}$ was studied. In [5], a family of sets approximately covers a set $U$ if it covers all but an $\varepsilon$ fraction of its volume. Bounds on the size of $\mathcal{F}^{\prime}$ which depend only on $\varepsilon$ and the fatness of the sets in $\mathcal{F}$ are presented. Although the problems studied in [5] differ from the ones studied in this work, one may find some resemblance between their proof techniques.

\subsection{Algorithmic Motivation}

Jie Gao and the authors of this work have recently used a variant of Theorem 1.2 in the design of an efficient approximation algorithm for clustering [9]. Approximation algorithms lend themselves naturally to the notion of $\varepsilon$ contraction and expansion because a quantified slackness of $\varepsilon$ is allowed.

More specifically, in [9] we prove a variant of Theorem 1.2 for sets that consist of unions of axis-parallel slabs (which is a special case of Conjecture 1.1). Our theorem is then applied in the design of an efficient dynamic data structure which manages the intersection of such sets. The data structure, in turn, is used as a key element in a $(1+\varepsilon)$-approximation algorithm for the $k$-center clustering of incomplete data. It is plausible that the theorems and definitions presented in this paper will find algorithmic applications other than those presented in [9].

A well-known application of Helly's theorem $[4,18,19]$ is in the theory of uniform approximation: Let $X$ be any finite set, let $F$ be a $d$-dimensional vector space of functions $F: X \rightarrow \mathbb{R}$, let $g: X \rightarrow \mathbb{R}$, and let $r$ be a nonnegative function on $X$. If for every $x_{1}, \ldots, x_{d+1} \in X$, there is an $f \in F$ such that $\left|f\left(x_{i}\right)-g\left(x_{i}\right)\right| \leqslant r\left(x_{i}\right)$ for all $1 \leqslant i \leqslant d+1$, then there is an $f \in F$ such that $|f(x)-g(x)| \leqslant r(x)$ for all $x \in X$. The contrapositive is that there is a short witness for inapproximability: if $g$ cannot be approximated within the function $r$ on $X$, then there are some $d+1$ points at which it already cannot be approximated within $r$.

In case $g$ is approximable within $r$, Theorem 1.1 can be applied in a similar spirit, and we obtain a short witness tightly constraining the set of approximations. Specifically: for $X, F, d$, and $r$ as above and for $\varepsilon>0$, there is for every $g: X \rightarrow \mathbb{R}$ a finite set $Y \subseteq X$ of cardinality $|Y| \leqslant s(d, \varepsilon)$ such that if $|f(x)-g(x)| \leqslant(1-\varepsilon) r(x)$ for all $x \in Y$, then $|f(x)-g(x)| \leqslant r(x)$ for all $x \in X$. (Apply Theorem 1.1 with $C_{x}=\{f:|f(x)-g(x)| \leqslant r(x)\}, \mathcal{S}=\left\{C_{x}\right\}_{x \in X}$, and $\left.\mathcal{S}^{\prime}=\left\{C_{x}\right\}_{x \in Y}.\right)$

\subsection{Proof Techniques}

In Theorem 1.1, we wish to find a small witness for the intersection $A=\bigcap_{i} C_{i}$ of elements in $\mathcal{S}=\left\{C_{i}\right\}$. Namely, we are interested in a subset $\mathcal{S}^{\prime}$ of $\mathcal{S}$ such that the intersection of the contracted elements in $\mathcal{S}^{\prime}$ is contained in $A$. Roughly speaking, we show that for any point $x$ on the boundary of $A$, there exists a set $C_{i} \in \mathcal{S}$ such that the contraction $C_{i}^{-\varepsilon}$ of $C_{i}$ does not include $x$ together with a significant portion of the boundary of $A$. Finding such sets $C_{i}$ iteratively, we are able to cover the boundary of $A$, resulting in the desired collection. As we are dealing with general convex sets, 


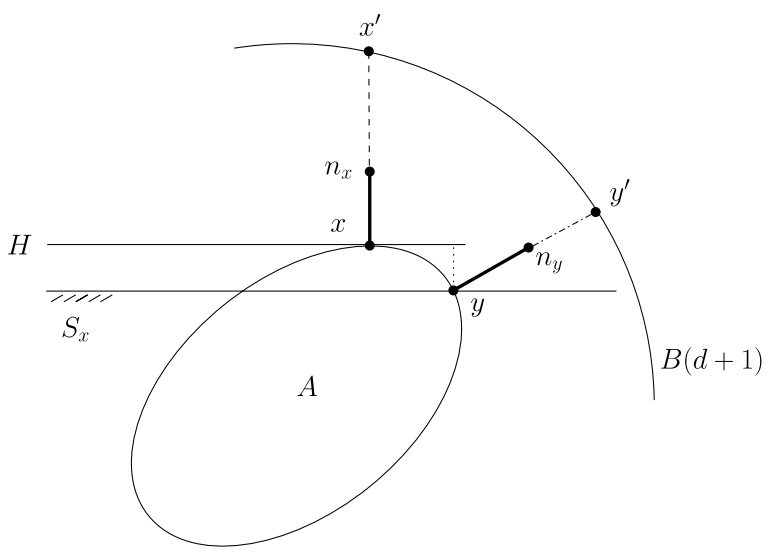

Fig. 3 An illustration of the proof of Theorem 1.1. Here $x$ is on the boundary of $A, H$ is the hyperplane tangent to $A$ at $x, n_{x}$ is of distance 1 from $x$ in the direction of the normal to $H$, and $x^{\prime}$ is the intersection of the boundary of $\mathcal{B}(d+1)$ and the extension of the line passing through $x$ and $n_{x}$. We denote by $S_{x}=S_{n_{x}}$ the half-space corresponding to $x$. The point $y$ is in $S_{x} \cap \partial A$, and both $n_{y}$ and $y^{\prime}$ are defined as above (with respect to $y$ ). If the distance between $H$ and the point $y$ is at least $\varepsilon$, then the distance between $x^{\prime}$ and $y^{\prime}$ is greater than $\sqrt{\varepsilon}$

giving a precise quantification of our progress towards covering the boundary of $A$ is the primary technical difficulty in our proof. The proof of Theorem 1.1 somewhat resembles the proof of Dudley for convex shape approximation by a polytope with few vertices [6].

In Theorem 1.2 we wish to find a small witness for the intersection $A$ of $\mathcal{S}=\left\{C_{i}\right\}$ when the sets $C_{i}$ are not necessarily convex; rather they are the union of $k$ convex sets. In a nutshell, the theorem is proven by induction on $k$, where Theorem 1.1 acts as the base case. The inductive step strongly uses the $f$-fatness of the sets in $C_{i}$.

\subsection{Organization}

The rest of the paper is organized as follows. In Sect. 2 we prove Theorems 1.1 and 1.2. In Sect. 3 we refine the analysis of Theorem 1.2 and obtain tighter bounds on the Helly number for the special case of sets in $\mathbb{R}$ (i.e., for the case $d=1$ ). In Sect. 4 we present lower bounds on the Helly numbers obtained throughout the paper. Finally, in Sect. 5 we present a few equivalent definitions to our notion of contraction and expansion, and prove some basic properties of these operations.

\section{Proof of our Helly Type Theorems}

We now present the proofs of Theorems 1.1 and 1.2. The reader may find it helpful to follow the proof of Theorem 1.1 using Fig. 3. The proof of Theorem 1.1 somewhat resembles the proof of Dudley for convex shape approximation by a polytope with few vertices [6]. 


\subsection{Proof of Theorem 1.1}

Let $A=\bigcap_{C \in \mathcal{S}}$. If $A$ is empty, then our theorem follows from Helly's theorem (without the need for contractions). Otherwise assume that $A$ has full dimension. We will address the case in which $A$ has dimension $\leqslant d-1$ at the end of the proof. By John's Theorem [13], there exists a point $p \in A$ and an ellipsoid $E$ such that $E \subseteq A \subseteq d(E-p)+p$. Here we use the fact that $A$ is bounded. Recalling that the hypotheses and conclusion of our theorem commute with affine transformations, we may assume w.l.o.g. that $p$ is the origin and that $E$ is the unit ball.

Let $\mathcal{B}(r)$ be the ball centered at the origin with radius $r$. By the assumptions above, $\mathcal{B}(1) \subseteq A \subseteq \mathcal{B}(d)$. We will now iteratively find the subset $\mathcal{S}^{\prime}$ stated in the theorem. Before we start, we give a rough overview of our iterative procedure. We start out with an empty family $\mathcal{S}^{\prime}$. Let $x$ be a point on the boundary of $A$. We show the existence of a set $C_{x} \in \mathcal{S}$ such that the contraction $C_{x}^{-\varepsilon}$ does not include $x$ together with any other point in $A$ within distance $\varepsilon$ from $x$. Adding $C_{x}$ to $\mathcal{S}^{\prime}$, we now seek a point $y$ on the boundary of $A$ that is included in $\bigcap_{C \in \mathcal{S}^{\prime}} C^{-\varepsilon}=C_{x}^{-\varepsilon}$. We add the corresponding set $C_{y}$ to $\mathcal{S}^{\prime}$ and stress that $y$ is far from the point $x$. We continue this process iteratively, and in each step we seek a point $z$ on the boundary of $A$ that is included in the set $\bigcap_{C \in \mathcal{S}^{\prime}} C^{-\varepsilon}$. If such a point $z$ is found, we add the corresponding set $C_{z}$ to $\mathcal{S}^{\prime}$ and progress in an additional iteration. If no such $z$ exists, we terminate our procedure. To bound the size of the resulting set $\mathcal{S}^{\prime}$, we notice that for any two sets $C_{x}$ and $C_{y}$ in $\mathcal{S}^{\prime}$, the corresponding points $x$ and $y$ on the boundary of $A$ are far apart. This fact allows us to use a certain packing argument that bounds the size of $\mathcal{S}^{\prime}$. Our packing argument will involve the lifting of the points $x$ on the boundary of $A$ onto the boundary of the ball $\mathcal{B}(d+1)$.

We start with the following technical lemma whose proof is based on the proof of a similar lemma presented in [10, p. 324]. The proof is illustrated in Fig. 3. For a point $x$ on the boundary of $A$, let $\mathcal{N}_{x}$ be the set consisting of points $n$ at distance 1 from $x$ in the direction of the normal to a tangent hyperplane of $A$ at $x$ (directed away from $A$ ). Notice that $\left|\mathcal{N}_{x}\right|$ may be larger than 1 (as there may be many tangent hyperplanes to $A$ at $x$ if $A$ is not smooth at $x$ ).

Lemma 2.1 Let $\varepsilon \in[0,1]$. Let $x$ and $y$ be two points on the boundary of $A$. Let $n_{x} \in \mathcal{N}_{x}$ and $n_{y} \in \mathcal{N}_{y}$. If the distance between $y$ and the hyperplane tangent to $A$ at $x$ with normal $n_{x}-x$ is at least $\varepsilon$, then $\left\|n_{x}-n_{y}\right\| \geqslant \sqrt{\varepsilon}$.

Proof Let $\theta$ be the angle between $n_{x}-x$ and $n_{y}-y$. Consider the plane $P$ including the points $x, y$, and $n_{x}$. It may be the case that $n_{y}$ does not lie on this plane. Let $n_{y}^{\prime}$ be the projection of $n_{y}$ onto $P$. Let $\theta^{\prime}$ be the angle between $n_{x}-x$ and $n_{y}^{\prime}-y$. Notice that $\theta^{\prime} \leqslant \theta$. Let $z$ be the point closest to $y$ on the hyperplane tangent to $A$ at $x$ (with normal $n_{x}-x$ ). Namely, $|y z| \geqslant \varepsilon$. We first assume that $\theta<\pi / 2$. Let $f$ be the point on $P$ in the intersection of the hyperplane tangent to $A$ at $x$ (with normal $n_{x}-x$ ) and the hyperplane tangent to $A$ at $y$ (with normal $n_{y}-y$ ). It holds that $\|x-y\| \geqslant\|y-f\|=|y z| / \sin \theta^{\prime}$. We now consider two cases. If $\sin \theta^{\prime} \leqslant \sqrt{\varepsilon}$, then $\left\|n_{x}-n_{y}\right\| \geqslant\|x-y\| \geqslant|y z| / \sin \theta^{\prime} \geqslant \sqrt{\varepsilon}$. The first inequality follows from the convexity of $A$ (namely, by the fact that $\left\langle n_{x}-x, x-y\right\rangle \geqslant 0$ and $\left\langle n_{y}-y, y-x\right\rangle \geqslant 0$ 
and by squaring $\left.\left\|n_{x}-n_{y}\right\|=\left\|x+\left(n_{x}-x\right)-y-\left(n_{y}-y\right)\right\|\right)$. If $\sin \theta^{\prime}>\sqrt{\varepsilon}$, we show that $\left\|n_{x}-n_{y}\right\| \geqslant\left\|\left(n_{x}-x\right)-\left(n_{y}-y\right)\right\| \geqslant 2 \sin (\theta / 2) \geqslant \sin \theta \geqslant \sin \left(\theta^{\prime}\right)>\sqrt{\varepsilon}$. Here, the first inequality follows from the convexity of $A$ as above, and the second inequality follows from considering the triangle $y, n_{y},\left(y+n_{x}-x\right)$. Now, for the case $\theta \geqslant \pi / 2$, it is not hard to verify that $\left\|n_{x}-n_{y}\right\| \geqslant 1$.

We continue with a few additional definitions that are central to the remainder of the proof (the definitions and proof are illustrated in Fig. 3). Let $x$ be any point on the boundary of $A$. For a special point $n_{x} \in \mathcal{N}_{x}$ (to be implied in the upcoming Claim 2.1), let $s_{n_{x}-x}(A)$ be the minimal $d$-dimensional slab containing $A$ with normal $n_{x}-x$ (i.e., the boundary of $s_{n_{x}-x}(A)$ is tangent to $A$ at the point $x$ ). In what follows, to simplify notation, we denote $s_{n_{x}-x}(A)$ by $s_{x}(A)$. Let $S_{x}$ be the closed half-space that does not include $x$ whose boundary equals the boundary of $s_{x}^{-\varepsilon}(A)$ closest to $x$. Roughly speaking, we will now show that for any $x \in \partial A$ : (a) a significant portion of $\partial A$ is not in $S_{x}$, and (b) there is a set $C_{x} \in \mathcal{S}$ such that $C_{x}^{-\varepsilon} \subseteq S_{x}$. Thus, taking enough points $x$ and their corresponding sets $C_{x}$ to be in $\mathcal{S}^{\prime}$, we will cover $\partial A$ and conclude our proof.

Claim 2.1 Let $x$ be any point on the boundary of $A$. There exist a point $n_{x} \in \mathcal{N}_{x}$ and a set $C_{x} \in \mathcal{S}$ such that $C_{x}^{-\varepsilon} \subseteq S_{x}$. Here, $S_{x}$ is the closed half-space (that does not include $x$ ) defined by the boundary of $s_{n_{x}-x}^{-\varepsilon}(A)$ closest to $x$.

Proof As $x \in \partial A$, there exists $C \in \mathcal{S}$ such that $x \in \partial C$. Let $H$ be a support hyperplane to $C$ at $x$. As $A \subseteq C, A$ is also included in one of the two half-spaces defined by $H$, which implies that $H$ is tangent to $A$ at $x$.

Let $u \in S^{d-1}$ be the normal of $H$ (directed away from $A$ ). Let $n_{x}=x+u$. Now, we have that $C^{-\varepsilon} \subseteq s_{u}^{-\varepsilon}(C)$. So it is left to show that $s_{u}^{-\varepsilon}(C) \subseteq S_{x}$. It holds that (a) $A \subseteq C$, and thus $s_{u}(A) \subseteq s_{u}(C)$; and (b) the boundary of $s_{u}(A)$ which includes $x$ and the boundary of $s_{u}(C)$ which includes $x$ are both equal to $H$. Thus we may conclude by our definition of $S_{x}$ that $s_{u}^{-\varepsilon}(C) \subseteq S_{x}$.

Let $x$ be any point on the boundary of $A$. Claim 2.1 specifies a special point $n_{x} \in \mathcal{N}_{x}$ and a special set $C_{x}$, both corresponding to $x$. From this point on in our proof, $n_{x}$ and $C_{x}$ will refer to this point and set, respectively. Moreover, the sets $s_{x}(A)$ and $S_{x}$ are defined according to this special $n_{x}$. We will now construct the set $\mathcal{S}^{\prime}$. Initially $\mathcal{S}^{\prime}$ is empty. Let $C_{x}$ be the set suggested in Claim 2.1 and add it to the set $\mathcal{S}^{\prime}$. It holds that $\bigcap_{C_{x} \in \mathcal{S}^{\prime}} C_{x}^{-\varepsilon} \subseteq \bigcap_{C_{x} \in \mathcal{S}^{\prime}} S_{x}$. In what follows we will add sets to $\mathcal{S}^{\prime}$ that will satisfy the above inclusion. Each set will be associated with a point on the boundary of $A$. We will show that after adding a sufficient number of specially chosen sets, the intersection $\bigcap_{C_{x} \in \mathcal{S}^{\prime}} S_{x}$ will be included in $A$, implying that $\bigcap_{C_{x} \in \mathcal{S}^{\prime}} C_{x}^{-\varepsilon} \subseteq A$.

Consider the ball $\mathcal{B}(d+1)$. By our discussion, it holds that for any point $x$ on the boundary of $A$, the distance between $x$ and the boundary of $\mathcal{B}(d+1)$ is at least 1 . For each point $x$ on the boundary of $A$, we will define a corresponding point $x^{\prime}$ on the boundary of $\mathcal{B}(d+1): x^{\prime}$ is the intersection of the boundary of $\mathcal{B}(d+1)$ and the line passing through $x$ and $n_{x}$ (in the direction opposite to $A$ ). We will call $x^{\prime}$ the projection of $x$ up to $\mathcal{B}(d+1)$. See Fig. 3 . 
We now return to the first point $x$ chosen and its corresponding set $C_{x}$ in $\mathcal{S}^{\prime}$. Let $y$ be any point on the boundary of $A$ that is included in $S_{x}$. If no such $y$ exists, then $A \cap S_{x}=\bigcap_{C \in \mathcal{S}} C \cap S_{x}=\emptyset$. By Helly's theorem there exist at most $d+1$ sets in $\mathcal{S}$ such that their intersection with $S_{x}$ is empty. Adding these sets to $\mathcal{S}^{\prime}$, we obtain $\bigcap_{C_{x} \in \mathcal{S}^{\prime}} C^{-\varepsilon}=\emptyset \subseteq A$. Otherwise:

Lemma 2.2 The distance between $y^{\prime}$ and $x^{\prime}$ must be at least $\sqrt{\varepsilon}$.

Proof Let $H$ be the tangent hyperplane at $x$ (with normal $n_{x}-x$ ). We first claim that the distance between $y$ and $H$ is at least $\varepsilon$. Indeed, this follows as $\mathcal{B}(1) \subseteq A \subseteq$ $s_{x}(A)$ and $y \in S_{x}$. Thus, by Lemma 2.1, the distance between $n_{x}$ and $n_{y}$ is at least $\sqrt{\varepsilon}$. Notice that $n_{x}$ is in $\mathcal{B}(d+1)$, thus $x^{\prime}$ is on the line passing through $x$ and $n_{x}$ after the point $n_{x}$. The same holds respectively for $y^{\prime}$. Namely, $x^{\prime}=n_{x}+\alpha\left(n_{x}-x\right)$ and $y^{\prime}=n_{y}+\beta\left(n_{y}-y\right)$ for some $\alpha>0$ and $\beta>0$. We would like to show that $\left\|x^{\prime}-y^{\prime}\right\| \geqslant\left\|n_{x}-n_{y}\right\|$. This follows from the fact that $\left\langle n_{x}-x, n_{x}-n_{y}\right\rangle \geqslant 0$ and $\left\langle n_{y}-y, n_{y}-n_{x}\right\rangle \geqslant 0$ (which in turn follows from the convexity of $A$ ). Specifically, $\left\|x^{\prime}-y^{\prime}\right\|^{2}=\left\|n_{x}+\alpha\left(n_{x}-x\right)-n_{y}-\beta\left(n_{y}-y\right)\right\|^{2}=\left\|n_{x}-n_{y}\right\|^{2}+\| \alpha\left(n_{x}-x\right)-$ $\beta\left(n_{y}-y\right)\left\|^{2}+2\left\langle\alpha\left(n_{x}-x\right), n_{x}-n_{y}\right\rangle+2\left\langle\beta\left(n_{y}-y\right), n_{y}-n_{x}\right\rangle \geqslant\right\| n_{x}-n_{y} \|^{2}$.

We are now ready to complete the proof of Theorem 1.1. After choosing $x$, we have shown that any $y \in S_{x}$ that remains on the boundary of $A$ has projection $y^{\prime}$ which is far from the projection $x^{\prime}$ of $x$. Now consider taking any such $y$ and adding its corresponding set $C_{y}$ as defined in Claim 2.1 to $\mathcal{S}^{\prime}$. If $(\partial A) \cap\left(\bigcap_{C_{x} \in \mathcal{S}^{\prime}} C^{-\varepsilon}\right)$ is empty, then either $\bigcap_{C_{x} \in \mathcal{S}^{\prime}} C^{-\varepsilon} \subset A$ and we are done, or $A \cap\left(\bigcap_{C_{x} \in \mathcal{S}^{\prime}} C^{-\varepsilon}\right)=\emptyset$. In the second case, we proceed as we did before by noticing that $\bigcap_{C \in \mathcal{S}} C \cap\left(\bigcap_{C_{x} \in \mathcal{S}^{\prime}} C^{-\varepsilon}\right)=\emptyset$. Hence, using Helly's theorem, we may add at most $d+1$ sets to $\mathcal{S}^{\prime}$ to obtain $\bigcap_{C \in \mathcal{S}^{\prime}} C^{-\varepsilon}=\emptyset \subseteq A$. Here we use the fact that for convex $C, C^{-\varepsilon}$ is also convex.

Otherwise, let $z$ be any point on the boundary of $A$ that is included in $\bigcap_{C_{x} \in \mathcal{S}^{\prime}} S_{x}$. By Lemma 2.2, $z^{\prime}$ is far from both $x^{\prime}$ and $y^{\prime}$. We now continue by adding to $\mathcal{S}^{\prime}$ the set $C_{z}$ corresponding to $z$.

Continuing this line of argument, it is not hard to verify that at any given stage in this process the set $\left\{x^{\prime} \mid C_{x} \in \mathcal{S}^{\prime}\right\}$ is a set of points on the boundary of $\mathcal{B}(d+1)$, and any two points in $\left\{x^{\prime} \mid C_{x} \in \mathcal{S}^{\prime}\right\}$ are of distance at least $\sqrt{\varepsilon}$. Standard packing arguments show that this implies that $\left|\mathcal{S}^{\prime}\right| \leqslant \frac{(c d)^{d}}{\varepsilon^{\frac{d}{2}}}$ for a universal constant $c>0$. This concludes our proof for sets $A$ of full dimension.

We now address the case in which $A$ does not have full dimension. Namely $A$ is included in a $k$-dimensional hyperplane $H$ for some $k \in\{0,1, \ldots, d-1\}$. Let $x$ be a point in $A$ which is not on the $k$-dimensional boundary of $A$. As mentioned in Claim 2.1, there exists a set $C$ such that $x \in \partial C$. If $C \subseteq H$, we may add $C$ to $\mathcal{S}^{\prime}$ and reduce the dimension of the problem. Otherwise, $C^{-\varepsilon} \cap A \subseteq C^{-\varepsilon} \cap H=\emptyset$, and we can add at most $d+1$ additional sets to $\mathcal{S}^{\prime}$ to obtain $\bigcap_{C \in \mathcal{S}^{\prime}} C^{-\varepsilon}=\emptyset \subseteq A$ (as done above).

\subsection{Proof of Theorem 1.2}

Recall that for $f \geqslant 1$, a convex set $C$ is $f$-fat if the ratio between the radii of the minimum radius ball containing $C$ and the maximum radius ball contained in $C$ is 
at most $f$ (see, e.g., [8]). Also, recall that for a set $C$ consisting of the union of $k$ convex sets $\left\{C_{1}, \ldots, C_{k}\right\}, C^{-\varepsilon} \triangleq \bigcup_{i=1}^{k} C_{i}^{-\varepsilon}$. For a convex set $C$, let $w(C)$ denote its width. Here $w(C)=\inf _{u \in S^{d-1}} w\left(s_{u}(C)\right)$, where the width $w\left(s_{u}(C)\right)$ of the $u$-slab $s_{u}(C)$ is defined to be the maximum length line segment in the direction $u$ contained in $s_{u}(C)$. As the width of $C$ is larger than twice the radius of the any ball contained in $C$, it follows that for $f$-fat sets $C, \operatorname{vol}(C)$ is at most the volume of a ball of radius $f w(C) / 2$. We now restate (an equivalent version of) Theorem 1.2 and present its proof.

Theorem 2.1 (Restatement of Theorem 1.2) Let $\mathcal{S}=\left\{C_{i}\right\}$ where each $C_{i}$ is the union of at most $k$ compact convex sets in $R^{d}$ each of which has fatness at most $f$. Let $I$ be any convex set. There exists a subset $\mathcal{S}^{\prime}$ of $\mathcal{S}$ of size at most $s(k, d, \varepsilon, f)=$ $k !\left(\frac{c^{k} d f^{k-1}}{\varepsilon^{k-\frac{1}{2}}}\right)^{d}$ such that $I \cap\left(\bigcap_{C \in \mathcal{S}^{\prime}} C^{-\varepsilon}\right) \subseteq I \cap\left(\bigcap_{C \in \mathcal{S}} C\right)$ (here $c>0$ is a universal constant).

Proof The proof is by induction on $k$. The base case, $k=1$, is implied by Theorem 1.1 . For general $k$, we will suppose that every $C_{i}$ consists of exactly $k$ nonempty sets $C_{i, 1}, \ldots, C_{i, k}$. This is true without loss of generality: simply duplicate one of the nonempty constituents. If some $C_{i}$ is empty, of course, the theorem follows trivially.

Recall that $w(A)$ is defined to be the width of a set $A$ in $\mathbb{R}^{d}$. For a collection $C_{i}$, let $w\left(C_{i}\right)=\max _{j} w\left(C_{i, j}\right)$. Let $w(\mathcal{S})=\inf _{i} w\left(C_{i}\right)$; we wish to pick an $i$ for which $w\left(C_{i}\right)=w(\mathcal{S})$. Strictly speaking, the infimum might not be achieved, but we will assume that $w\left(C_{1}\right)=w(\mathcal{S})$ (otherwise slight changes are to be made in the proof).

We include $C_{1}$ in our collection and achieve the required intersection separately within $I \cap C_{1, j}$ for each $1 \leqslant j \leqslant k$. Because $C_{1, j}$ is $f$-fat, it can be covered by at most $\left(\frac{4 f}{\varepsilon}\right)^{d}$ balls of radius $\frac{\varepsilon}{4} w(\mathcal{S})$. We achieve the required intersection separately within each of these balls. Let $\mathcal{B}$ be one of the balls.

Let $\mathcal{S}^{B}=\left\{C_{i} \mid \mathcal{B} \cap I \nsubseteq C_{i}\right\}$ and observe that $I \cap \mathcal{B} \cap\left(\bigcap_{\mathcal{S}^{B}} C_{i}\right)=I \cap \mathcal{B} \cap\left(\bigcap_{\mathcal{S}} C_{i}\right)$. For each $C_{i}$, form a collection $\hat{C}_{i}$ of $k-1$ sets by omitting from $C_{i}$ a set of greatest width (which without loss of generality we label $C_{i, 1}$ ).

Observe that $\hat{C}_{i}$ is a family of type $k-1$ and that $(I \cap \mathcal{B}) \cap\left(\bigcap_{\mathcal{S}^{B}} \hat{C}_{i}\right) \subseteq(I \cap \mathcal{B}) \cap$ $\left(\bigcap_{\mathcal{S}^{B}} C_{i}\right)$. So there is a set of indices $I^{B}$ of cardinality at most $s(k-1, d, \varepsilon, f)$ for which $(I \cap \mathcal{B}) \cap\left(\bigcap_{i \in I^{B}} \hat{C}_{i}^{-\varepsilon}\right) \subseteq(I \cap \mathcal{B}) \cap\left(\bigcap_{\mathcal{S}^{B}} C_{i}\right)$.

Now since $w\left(C_{i, 1}\right) \geqslant w(\mathcal{S})$, it is not hard to verify that $(I \cap B) \cap C_{i, 1}^{-\varepsilon}=\emptyset$ for every $i \in I_{B}$. This follows from the fact that (a) for any unit vector $u$, the width $w_{u}(C)$ of $s_{u}(C)$ is at least $w(\mathcal{S})$, and (b) $C_{i, 1}^{-\varepsilon}=\bigcap s_{u}^{-\varepsilon}\left(C_{i, 1}\right)$. Therefore

$$
\begin{aligned}
(I \cap \mathcal{B}) \cap\left(\bigcap_{i \in I^{B}}\left(C_{i}\right)^{-\varepsilon}\right) & =(I \cap \mathcal{B}) \cap\left(\bigcap_{I^{B}} \hat{C}_{i}^{-\varepsilon}\right) \subseteq(I \cap \mathcal{B}) \cap\left(\bigcap_{\mathcal{S}^{B}} C_{i}\right) \\
& =I \cap \mathcal{B} \cap\left(\bigcap_{\mathcal{S}} C_{i}\right) .
\end{aligned}
$$

Therefore $s(k, d, \varepsilon, f) \leqslant \frac{4^{d} k f^{d}}{\varepsilon^{d}} s(k-1, d, \varepsilon, f)$, which suffices to prove our assertion. 


\section{Improved Bounds for $d=1$}

In this section we improve on the bound for $s(k, d, \varepsilon, f)$ presented in Theorem 1.2 when $d=1$. Notice that convex sets $C \in \mathbb{R}$ are $f$-fat for $f=1$. Thus, for $d=1$, Theorem 1.2 implies a bound on $s(k, d, \varepsilon, f)=s(k, 1, \varepsilon)$ which is proportional to $\varepsilon^{-k}$. In what follows we present a proof implying a bound approximately proportional to $\varepsilon^{-\frac{k}{2}}$. The line of proof is very similar to that of Theorem 2.1.

Theorem 3.1 Let $\mathcal{S}=\left\{C_{i}\right\}$ where each $C_{i}$ is the union of at most $k(k=2 \ell$ or $k=2 \ell+1)$ compact convex sets in $\mathbb{R}$. Let $I$ be a convex set in $\mathbb{R}$. Then, there is a universal constant $c$ such that there is a subset $\mathcal{S}^{\prime}$ of $\mathcal{S}$ of size at most $s(2 \ell, 1, \varepsilon)=(c / \varepsilon)^{\ell} \log ^{\ell-1}(1 / \varepsilon)$ or $s(2 \ell+1,1, \varepsilon)=2(c / \varepsilon)^{\ell} \log ^{\ell}(1 / \varepsilon)$ such that $I \cap\left(\bigcap_{C \in \mathcal{S}^{\prime}} C^{-\varepsilon}\right) \subseteq I \cap\left(\bigcap_{C \in \mathcal{S}} C\right)$.

Proof The proof is by induction on $k$; there are two base cases: $k=0$, for which we may formally take $s(0,1, \varepsilon)=1$, and $k=1$, for which $s(1,1, \varepsilon)=2$ (a version of Helly's theorem in $\mathbb{R}$ ).

Write $C_{i}=\left\{\left[A_{i}^{j}, B_{i}^{j}\right]\right\}_{j \in\{1, \ldots, k\}}$. Set $\underline{A}(\mathcal{S})=\max _{C_{i} \in \mathcal{S}} \min _{j} A_{i}^{j}$ and $\bar{B}(\mathcal{S})=$ $\min _{C_{i} \in \mathcal{S}} \max _{j} B_{i}^{j}$. Use two elements of the family to form an intersection included in $[\underline{A}(\mathcal{S}), \bar{B}(\mathcal{S})]$, and let $[\alpha, \beta]=I \cap[\underline{A}(\mathcal{S}), \bar{B}(\mathcal{S})]$. By hypothesis, $[\alpha, \beta] \cap$ $\left(\bigcap_{C \in \mathcal{S}} C\right)=I \cap\left(\bigcap_{C \in \mathcal{S}} C\right)$. Observe that if a set $C_{i}$ does not contain some point $x \in[\alpha, \beta]$, then $C_{i}$ has an interval $\left[A_{i}^{j}, B_{i}^{j}\right]$ for which $A_{i}^{j} \leqslant \alpha$ and $B_{i}^{j}<x$, and another interval $\left[A_{i}^{j}, B_{i}^{j}\right]$ for which $x<A_{i}^{j}$ and $\beta \leqslant B_{i}^{j}$.

We show separately how to obtain the desired intersection in each of the four closed intervals (whose union is $[\alpha, \beta]$ ) that are demarcated by the five points $\alpha$, $\left(1-\frac{\varepsilon}{2}\right) \alpha+\frac{\varepsilon}{2} \beta,(1 / 2) \alpha+(1 / 2) \beta, \frac{\varepsilon}{2} \alpha+\left(1-\frac{\varepsilon}{2}\right) \beta, \beta$. The first and fourth intervals can be treated in the same way, as can the second and third, so we consider only the first and second.

The interval $\left[\alpha,\left(1-\frac{\varepsilon}{2}\right) \alpha+\frac{\varepsilon}{2} \beta\right]$ : Modify the collection $\mathcal{S}$ by (a) omitting sets $C_{i}$ that include the interval $\left[\alpha,\left(1-\frac{\varepsilon}{2}\right) \alpha+\frac{\varepsilon}{2} \beta\right]$ and (b) omitting from each $C_{i}$ every interval $\left[A_{i}^{j}, B_{i}^{j}\right]$ for which $B_{i}^{j} \geqslant \beta$. At least one interval is omitted from each $C_{i}$, so in the modified collection, every set is the union of at most $k-1$ intervals. By induction, $s(k-1,1, \varepsilon)$ members of $\mathcal{S}$ suffice (after contraction) in order to obtain the desired intersection with $\left[\alpha,\left(1-\frac{\varepsilon}{2}\right) \alpha+\frac{\varepsilon}{2} \beta\right]$. Restoring the omitted intervals of each of these members does not change this, because after contraction those are disjoint from $\left[\alpha,\left(1-\frac{\varepsilon}{2}\right) \alpha+\frac{\varepsilon}{2} \beta\right]$.

The interval $\left[\left(1-\frac{\varepsilon}{2}\right) \alpha+\frac{\varepsilon}{2} \beta,(1 / 2) \alpha+(1 / 2) \beta\right]$ : Partition this interval into the $t=\log _{1-\frac{\varepsilon}{2}} \varepsilon$ intervals demarcated by the following points (for simplicity, we assume that $\left.\left(1-\frac{\varepsilon}{2}\right)^{t}=\varepsilon\right)$ :

$$
\begin{aligned}
& x_{1}=\left(1-\frac{\varepsilon}{2}\right) \alpha+\frac{\varepsilon}{2} \beta, \\
& x_{2}=\left(1-\frac{\frac{\varepsilon}{2}}{1-\frac{\varepsilon}{2}}\right) \alpha+\frac{\frac{\varepsilon}{2}}{1-\frac{\varepsilon}{2}} \beta,
\end{aligned}
$$




$$
\begin{aligned}
x_{3} & =\left(1-\frac{\frac{\varepsilon}{2}}{\left(1-\frac{\varepsilon}{2}\right)^{2}}\right) \alpha+\frac{\frac{\varepsilon}{2}}{\left(1-\frac{\varepsilon}{2}\right)^{2}} \beta, \ldots, \\
x_{t+1} & =(1 / 2) \alpha+(1 / 2) \beta .
\end{aligned}
$$

Consider any one of these intervals, $\left[x_{m}, x_{m+1}\right]$. Form a collection $\mathcal{S}^{m}$ by omitting from $\mathcal{S}$ any set $C_{i}$ containing $\left[x_{m}, x_{m+1}\right]$. The collection $\mathcal{S}^{m}$ has the property that $\left[x_{m}, x_{m+1}\right] \cap\left(\bigcap_{C \in \mathcal{S}^{m}} C\right)=\left[x_{m}, x_{m+1}\right] \cap\left(\bigcap_{C \in \mathcal{S}} C\right)$. Moreover, each $C_{i}$ in $\mathcal{S}^{m}$ has at least one interval $\left[A_{i}^{j}, B_{i}^{j}\right]$ for which $A_{i}^{j} \leqslant \alpha$ and $B_{i}^{j}<x_{m+1}$, and a different interval $\left[A_{i}^{j}, B_{i}^{j}\right]$ for which $A_{i}^{j}>x_{m}$ and $B_{i}^{j} \geqslant \beta$. Modify $\mathcal{S}^{m}$ by omitting from each $C_{i}$ any interval satisfying one of these conditions. In the modified collection, every set is the union of at most $k-2$ intervals. By induction, $s(k-2,1, \varepsilon)$ members of the modified $\mathcal{S}^{m}$ suffice (after contraction) in order to obtain the desired intersection with $\left[x_{m}, x_{m+1}\right]$. Restoring the omitted intervals of each of these members does not change this, because after contraction those intervals are disjoint from $\left[x_{m}, x_{m+1}\right]$.

We have shown that for $k \geqslant 2, s(k, 1, \varepsilon) \leqslant 2+2 s(k-1,1, \varepsilon)+2\left(\log _{1-\frac{\varepsilon}{2}} \varepsilon\right) s(k-$ $2,1, \varepsilon)$. This implies the stated bound for a suitable value of $c$.

\section{Lower Bounds}

We now sketch some lower bounds on the size of $s(d, \varepsilon)$ and $s(k, 1, \varepsilon)$ from Theorems 1.1 and 3.1, respectively. Roughly speaking, we show in these cases that our analysis as a function of $\varepsilon$ is nearly tight. For Theorem 1.1, consider the set system $\mathcal{S}=C_{u}$ indexed by all unit vectors $u \in S^{d-1} . C_{u}$ is defined to be the centrally symmetric $d$-dimensional slab around the origin, with width 2 and normal $u$. The intersection $\bigcap_{C \in \mathcal{S}} C$ is the unit ball $\mathcal{B}$. Every contracted set $C_{u}^{-\varepsilon}$ is the centrally symmetric $d$-dimensional slab around the origin with width $2-2 \varepsilon$. Standard calculations show that $\mathcal{B} \backslash C_{u}^{-\varepsilon}$ consists of two caps, each of area at most $(2 \varepsilon)^{\frac{d-1}{2}}$ times the surface area of $\mathcal{B}$. Thus, a set $\mathcal{S}^{\prime}$ of at least $\simeq(2 \varepsilon)^{-\frac{d-1}{2}}$ sets $C_{u}$ are required to obtain $\bigcap_{C \in \mathcal{S}^{\prime}} \subseteq \mathcal{B}$.

Following the proof of Theorem 3.1, a lower bound of $\left(\frac{1}{c \varepsilon}\right)^{\left\lfloor\frac{k}{2}\right\rfloor}$ for $s(k, 1, \varepsilon)$ can be obtained (for a universal constant $c$ ). Specifically (using the notation of Theorem 3.1), for $\alpha=0$ and $\beta=1$, we sketch our proof. For $i=0, \ldots, \frac{1}{2 \varepsilon}-1$, consider the intervals $[2 i \varepsilon, 2(i+1) \varepsilon]$. We define a set system $\mathcal{S}_{i}$ corresponding to each interval inductively. The resulting family $\mathcal{S}$ will be the union of the set systems $\mathcal{S}_{i}$ and will have empty intersection. By our construction, after contraction, all sets in $\mathcal{S}_{i}$ will include all but the $i$ th interval. For $i=1, \ldots, \frac{1}{2 \varepsilon}-2$, let $x=2 i \varepsilon$ and $y=2(i+1) \varepsilon$. The required set system $\mathcal{S}_{i}$ for $[x, y]$ will consists of sets $C=C_{1} \cup \cdots \cup C_{k}$ which all have the following structure: $C_{1}=\left[-\frac{x \varepsilon}{2-2 \varepsilon}, x+\frac{x \varepsilon}{2-2 \varepsilon}\right], C_{2}=\left[y-\frac{(1-y) \varepsilon}{2-2 \varepsilon}, 1+\frac{(1-y) \varepsilon}{2-2 \varepsilon}\right]$ (notice that $C_{1}^{-\varepsilon}=[0, x]$ and $C_{2}^{-\varepsilon}=[y, 1]$ ), and $C_{3}, \ldots, C_{k}$ are taken from a family $\hat{\mathcal{S}}_{i}$ constructed inductively. Each element $\hat{C}$ in the family $\hat{\mathcal{S}}_{i}$ is the union of $k-2$ convex sets, all included in the interval $\left[x+\frac{x \varepsilon}{2-2 \varepsilon}, y-\frac{(1-y) \varepsilon}{2-2 \varepsilon}\right]$. The inductive assumption is that the family of sets in $\hat{\mathcal{S}}_{i}$ has empty intersection, and any subset of 
them of size less than $\left(\frac{1}{c \varepsilon}\right)^{\left\lfloor\frac{k-2}{2}\right\rfloor}$ will not have empty intersection even after the sets are contracted. The base of the induction is either the set system consisting of an empty set $(k=0)$ or a set system consisting of two disjoint intervals $(k=1)$. For $i=0$ or $i=\frac{1}{2 \varepsilon}-1$, the set system $\mathcal{S}_{i}$ is constructed analogously. The set system $\mathcal{S}$ consists of all sets systems $\mathcal{S}_{i}$ defined above. It is not hard to verify that to obtain a subset $\mathcal{S}^{\prime}$ such that $\bigcap_{C \in \mathcal{S}^{\prime}} C^{-\varepsilon}=\emptyset$, for the $i$ th interval defined above, we need to use sets $C$ corresponding to $\mathcal{S}_{i}$.

\section{Contraction and Expansion of Convex Sets: Properties and Equivalent Definitions}

In the upcoming sections, we elaborate on the properties of $C^{\varepsilon}$. Specifically, in Sects. 5.1 and 5.2 we discuss a few additional natural definitions for contraction and expansion. Some of these definitions are equivalent to the one presented in the Introduction (Sect. 5.1) and others differ but are related (Sect. 5.2). As we will see in Sect. 6, these equivalent definitions will help us to extend Theorems 1.1 and 1.2 to the case of set systems $\mathcal{S}$ with unbounded sets $C$. It is not hard to verify that $\|\cdot\|_{C}$ as defined in Definition 1.1 is not necessarily a norm, even in cases where there is an obvious point in $C$ to try placing the origin. Primarily, it is not hard to verify that for certain $C$, such as triangles, the value of $\|x\|_{C}$ is strictly positive on $\mathbb{R}^{d}$. Nevertheless, we start by noticing that $\|\cdot\|_{C}$ is convex.

Claim 5.1 (Convexity of $\|\cdot\|_{C}$ ) For a convex set $C$, points $x_{1}$ and $x_{2}$ in $\mathbb{R}^{d}$, and $\lambda \in[0,1]$, it holds that $\left\|\lambda x_{1}+(1-\lambda) x_{2}\right\|_{C} \leqslant \lambda\left\|x_{1}\right\|_{C}+(1-\lambda)\left\|x_{2}\right\|_{C}$.

Proof Let $\left\|x_{1}\right\|_{C}=1+\varepsilon_{1}$ and $\left\|x_{2}\right\|_{C}=1+\varepsilon_{2}$. For $i=1,2$, this implies that $x_{i} \in C^{\varepsilon_{i}}$, which in turn implies for every $u \in S^{d-1}$ that $x_{i} \in s_{u}^{\varepsilon_{i}}(C)$. As $s_{u}(C)$ is a slab, it is not hard to verify that the above implies that $\lambda x_{1}+(1-\lambda) x_{2} \in s_{u}^{\lambda \varepsilon_{1}+(1-\lambda) \varepsilon_{2}}(C)$. We conclude that $\lambda x_{1}+(1-\lambda) x_{2} \in C^{\lambda \varepsilon_{1}+(1-\lambda) \varepsilon_{2}}$, which suffices to prove our assertion.

\subsection{Equivalent Definitions}

We now present some definitions equivalent to Definition 1.1. For a set $C \subseteq \mathbb{R}^{d}$, let $\partial C$ denote its boundary with respect to $\mathbb{R}^{d}$. For $\varepsilon>0$, let $\partial_{\varepsilon} C=\partial C+\varepsilon(C-C)$.

Consider again a centrally symmetric convex set $C$ centered at $p$ and let $x$ be a point outside $C$. Consider the line $\ell$ passing through $x$ and $p$. This line and its intersection with $C$ define the value $\|x\|_{C}$. Namely, let $z$ be the intersection of $\ell$ and the boundary of $C$ closest to $x$, and let $y$ be the intersection of $\ell$ and the boundary of $C$ furthest from $x$ (see Figs. 1 and 4). The value of $\|x\|_{C}$ as defined previously is a function of the distance $|x z|$ and the distance $|x y|$. Specifically, let $r=|x z| /|x y|$. It is not hard to verify that $\|x\|_{C}=\frac{|p x|}{|p z|}=\frac{1+r}{1-r}$. Similarly, for $x \in C$ and $y$ and $z$ as defined previously, it can be verified that $\|x\|_{C}=\frac{|p x|}{|p z|}=\frac{1-r}{1+r}$. This leads to the following definition for general convex sets. 


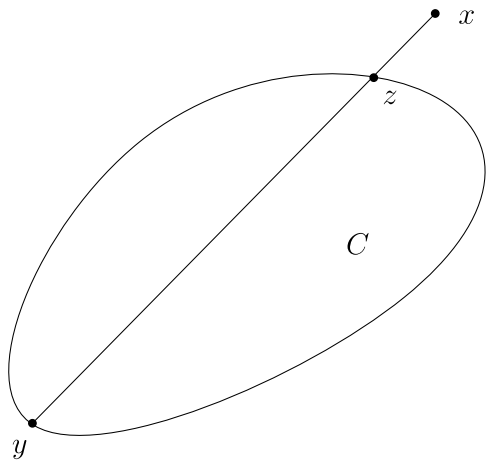

(a)

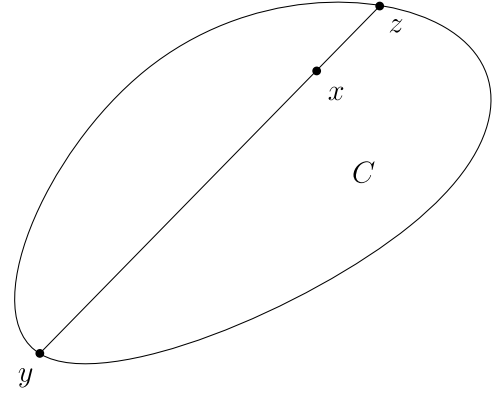

(b)

Fig. 4 An illustration of the definition of $C^{(\varepsilon)}$. In (a), for $\varepsilon>0, x \in C^{(\varepsilon)}$ iff there exist $y, z \in \partial C$ as above such that the ratio between $|x z|$ and $|x y|$ is at most $\frac{\varepsilon}{2+\varepsilon}$. In that case, $r(x, C) \leqslant \frac{\varepsilon}{2+\varepsilon}$, which implies that $n_{C}(x)=\frac{1+r(x, C)}{1-r(x, C)} \leqslant 1+\varepsilon$. Similarly, in (b), for $\varepsilon<0, x \in C^{(\varepsilon)}$ iff for all $y, z \in \partial C$ as above, the ratio between $|x z|$ and $|x y|$ is at least $\frac{-\varepsilon}{2+\varepsilon}$. In that case, $r(x, C) \leqslant \frac{\varepsilon}{2+\varepsilon}$, which implies that $n_{C}(x)=\frac{1+r(x, C)}{1-r(x, C)} \leqslant 1+\varepsilon$

For $C$ that is compact and convex, let $r(x, C) \in[-1,1]$ be the closest point to 0 in the closure of the set

$$
\left\{\frac{\langle z-x, y-x\rangle}{\langle y-x, y-x\rangle}\right\}_{z \in \partial C, y \in \partial C x, y, z \text { colinear }} .
$$

Let $n_{C}(x)=\frac{1+r(x, C)}{1-r(x, C)}$. Let $C^{(\varepsilon)}=\left\{x \mid n_{C}(x) \leqslant 1+\varepsilon\right\}$.

A few remarks are in order. Notice that $n_{C}(x)$ is greater than 1 for $x \notin C$ and less than or equal to 1 for $x \in C$. Moreover, $n_{C}(x)=1$ iff $x \in \partial C$. This follows from the definition of $r(x, C)$, which is negative for $x \in C \backslash \partial C$, has value 0 for $x \in \partial C$ and positive for $x \notin C$. As an example, for the interval $C=[-1,1]$, it holds that $C^{(\varepsilon)}=[-1-\varepsilon, 1+\varepsilon]$.

Theorem 5.1 (Equivalent definitions) Let $\varepsilon \geqslant 0$. Let $\partial_{\varepsilon} C=\partial C+\varepsilon(C-C)$.

(i) $C^{\varepsilon} \triangleq \bigcap_{u} s_{u}^{\varepsilon}(C)=C^{(\varepsilon)}=C \cup \partial_{\frac{\varepsilon}{2}} C$.

(ii) $C^{-\varepsilon} \triangleq \bigcap_{u} s_{u}^{-\varepsilon}(C)=C^{(-\varepsilon)}=C \backslash\left(\partial_{\frac{\varepsilon}{2}} C\right)^{\circ}$.

Here $(A)^{\circ}$ denotes the interior of $A$.

Proof As a warm up exercise, it is not hard to verify that the assertions hold if $C$ is centrally symmetric. We now present the proof for general $C$. First notice that for $\varepsilon \geqslant 0, x \in C^{(\varepsilon)}$ iff $r(x, C) \leqslant \frac{\varepsilon}{2+\varepsilon}$, and $x \in C^{(-\varepsilon)}$ iff $r(x, C) \leqslant \frac{-\varepsilon}{2-\varepsilon}$. These inequalities will be used throughout the following proof. Moreover, the set $C$ is included in all the expressions in (i) and includes all expressions in (ii). Hence, to prove (i), it suffices to show that (a) $x \in \bigcap_{u} s_{u}^{\varepsilon}(C) \backslash C \Rightarrow x \in C^{(\varepsilon)}$, (b) $x \in C^{(\varepsilon)} \backslash C \Rightarrow x \in C \cup \partial_{\frac{\varepsilon}{2}} C$, 
and (c) $x \in \partial_{\frac{\varepsilon}{2}} C \backslash C \Rightarrow x \in \bigcap_{u} s_{u}^{\varepsilon}(C)$. An analogous series of inclusions for (ii) assuming $x \in C$ also suffices.

(i): Let $x \in \bigcap s_{u}^{\varepsilon}(C) \backslash C$. We first show that $x \in C^{(\varepsilon)}$ by proving that $x \in$ $\bigcap s_{u}\left(C^{(\varepsilon)}\right)$. This suffices as it is not hard to verify that $C^{(\varepsilon)}$ is convex (this follows directly from its definition) and thus $\bigcap s_{u}\left(C^{(\varepsilon)}\right)=C^{(\varepsilon)}$. Consider a vector $u \in S^{d-1}$ and any $x \in s_{u}^{\varepsilon}(C)$. We now show that $x \in s_{u}\left(C^{(\varepsilon)}\right)$. If $x \in s_{u}(C)$, then clearly $x \in s_{u}\left(C^{(\varepsilon)}\right)$. Otherwise, let $y^{\prime}, z^{\prime}$ be the points on $\partial C$ that define $s_{u}(C)$ (take $z^{\prime}$ to be closer to $x$ than $y^{\prime}$ once all three are projected on $u$ ). Consider the line $\ell$ passing through $x$, in the direction $y^{\prime}-z^{\prime}$. Let $y, z$ be the intersection points of $\ell$ and the boundary of $s_{u}(C)$ (take $z$ to be closer to $x$ ). As $x \in s_{u}^{\varepsilon}(C) \backslash s_{u}(C)$, it holds that $|x z| /|x y|=\frac{\varepsilon^{\prime}}{2+\varepsilon^{\prime}}$ for some $\varepsilon^{\prime} \leqslant \varepsilon$. Let $x^{\prime}$ be on the line passing through $y^{\prime}$ and $z^{\prime}$ such that $\left|x^{\prime} z^{\prime}\right| /\left|x^{\prime} y^{\prime}\right|=\frac{\varepsilon^{\prime}}{2+\varepsilon^{\prime}}$. It holds that $x^{\prime} \in C^{(\varepsilon)} \subseteq s_{u}\left(C^{(\varepsilon)}\right)$. By our construction $x^{\prime}-x$ is orthogonal to $u$, thus $x \in s_{u}\left(C^{(\varepsilon)}\right)$.

Now let $x \in C^{(\varepsilon)} \backslash C$. Let $u \in S^{d-1}$ be any direction. Consider the line $\ell_{u}$ passing through $x$ in the direction of $u$. Let $z_{u}$ be the intersection point of $\ell_{u}$ and $C$ closest to $x$, and let $y_{u}$ be the intersection point farthest from $x$ (if such exists). It holds that $r(x, C)=\min _{u}\left|x z_{u}\right| /\left|x y_{u}\right| \leqslant \frac{\varepsilon}{2+\varepsilon}$. Let $r(x, C)$ be obtained at $u$, and denote $z=z_{u}$, $y=y_{u}$. As $z-y \in C-C$, it holds that $x \in z+\frac{\varepsilon}{2}(C-C) \subseteq C \cup \partial_{\frac{\varepsilon}{2}} C$.

Finally, consider $x \in \partial_{\frac{\varepsilon}{2}} C \backslash C$. This implies that $x=c+\frac{\varepsilon^{\prime}}{2}(y-z)$ for $\varepsilon^{\prime} \leqslant \varepsilon$, $y, z \in C$ and $c$ on $\partial C$. Let $u \in S^{d-1}$; we will show that $x \in s_{u}^{\varepsilon}(C)$. If $x \in s_{u}(C)$, then we are done. Let $\ell$ be the line passing through $x$ in the direction $y-z$ and intersecting $s_{u}(C)$ at $z^{\prime}$ and $y^{\prime}$. It holds that $\left|z^{\prime} y^{\prime}\right| \geqslant|z y|$. As $z^{\prime} y^{\prime} \subseteq s_{u}(C)$ and $c \in s_{u}(C)$, we conclude that $x \in s_{u}^{\varepsilon}(C)$.

(ii): Assume that $x \in C$ but $x \notin C^{(-\varepsilon)}$. Let $z$ and $y$ be in $\partial C$ such that $z, x, y$ are co-linear and $|z x| /|x y|<\frac{\varepsilon}{2-\varepsilon}$. Let $u$ be the normal to the hyperplane tangent at $z$ (directed away from $C$ ) and consider $s_{u}(C)$. Let $y^{\prime}$ be the point "opposite" to $z$ on the boundary of $s_{u}(C)$ (namely $y^{\prime}$ is obtained by going in direction $-u$ from $z$ until we leave the set $\left.s_{u}(C)\right)$. Let $x^{\prime}$ be the projection of $x$ to the line $z y^{\prime}$. It now holds that $\left|z x^{\prime}\right| /\left|y^{\prime} x^{\prime}\right| \leqslant|z x| /|x y|<\frac{\varepsilon}{2-\varepsilon}$. This implies that $x \notin s_{u}^{-\varepsilon}(C)$.

Assume that $x \in C$ but $x \notin C \backslash\left(\partial_{\frac{\varepsilon}{2}} C\right)^{\circ}$. Let $y$ and $z$ be in $C$ and $c \in \partial C$ such that $|x c|<\frac{\varepsilon}{2}|y z|$. Assume that $c-x$ is in the same direction as $z-y$. Consider the segment originating at $y$, passing through $x$ and terminating on $\partial C$ at $z^{\prime}$. We show that $\left|z^{\prime} x\right|<\frac{\varepsilon}{2}\left|y z^{\prime}\right|$. Let $\alpha$ be the intersection of the line passing through $z, c$ and the line passing through $y, x$ (here we assume that the points $z, y, x, c$ are not all on the same line; otherwise a similar and simpler proof may be given). Notice that the point $\alpha$ is not in $C^{o}$ (follows from the convexity of $C$ ). It holds that $\frac{z^{\prime} x}{z^{\prime} y} \leqslant \frac{\alpha x}{\alpha y}=\frac{c x}{z y}<\frac{\varepsilon}{2}$. We conclude that $x \notin C^{(-\varepsilon)}$.

Finally, assume that $x \in C$ but $x \notin s_{u}^{-\varepsilon}(C)$ for some $u$. Let $y$ and $z$ be the tangent points in $\partial C$ defining $s_{u}(C)$, and let $H_{y}$ and $H_{z}$ be the corresponding hyperplanes. Assume that $z$ is closer to $x$ than $y$ when all three points are projected onto the direction $u$. Consider the segment originating at $y$, passing through $x$, and terminating at $z^{\prime} \in \partial C$. We now show that $\left|x z^{\prime}\right|<\frac{\varepsilon}{2-\varepsilon}|x y|$, which implies that $x \notin C \backslash \partial \frac{\varepsilon}{2} C$. This follows by considering the triangle $y z^{\prime} \alpha$ where $\alpha$ is the projection of $z^{\prime}$ onto $H_{y}$. In this triangle, let $x^{\prime}$ be the projection of $x$ onto $z^{\prime} \alpha$. By our assumption, $\left|x^{\prime} z^{\prime}\right| /\left|x^{\prime} \alpha\right|<$ 
$\frac{\varepsilon}{2-\varepsilon}$. This implies that $\left|z^{\prime} x\right| /|x y|<\frac{\varepsilon}{2-\varepsilon}$, which in turn implies $x=z^{\prime}+\frac{\varepsilon^{\prime}}{2}\left(y-z^{\prime}\right)$ for $\varepsilon^{\prime}<\varepsilon$.

Remark 5.1 In the proofs that follow we will use the equivalence stated in Theorem 5.1 without referring explicitly to Theorem 5.1.

\subsection{Contraction and Expansion w.r.t. Points $p \in C$}

For a general convex set $C$, one may consider an alternative definition for $\|x\|_{C}$ and $C^{\varepsilon}$ in which the contraction and expansion are done with respect to a center point $p$ in $C$. Namely, as in the case of centrally symmetric sets, for $p \in C$, define $\|x\|_{(C, p)}=$ $\inf \left\{r>0 \mid \frac{x-p}{r}+p \in C\right\}$ and $C_{p}^{\varepsilon}=\left\{x \mid\|x\|_{(C, p)} \leqslant 1+\varepsilon\right\}$. Clearly, as this definition depends on the point $p$ chosen, it is not equivalent to our original definition given in Definition 1.1. For this reason, we prefer to use our original definition which depends solely on the set $C$.

Nevertheless, for specific values of $p$, one can find connections between our original definition and that given above. For $\alpha \geqslant 1$, a point $p \in C$ is referred to as $\alpha$-central if for any line $\ell$ passing through $p$, the distance between $p$ and the two intersection points of $\ell$ with $\partial C, z$ and $y$, satisfy $\frac{|z p|}{|y p|} \in\left[\frac{1}{\alpha}, \alpha\right]$. For example, if $C$ includes a point $p$ which is 1 -central (i.e., $\alpha=1$ ), then $C$ is centrally symmetric. Also, as we show below, the center of mass of $C$ is a " $d$-central" point. The same holds for points implied by John's theorem [13], which states the existence of an ellipsoid $E$ centered at $p \in C$ such that $E \subseteq C \subseteq d(E-p)+p$.

Lemma 5.1 Let $\alpha \geqslant 1$. Let $p$ be an $\alpha$-central point of $C$. For $\varepsilon \geqslant 0$, it holds that $C_{p}^{\frac{(1+1 / \alpha) \varepsilon}{2}} \stackrel{(a)}{\subseteq} C^{\varepsilon} \stackrel{(b)}{\subseteq} C_{p}^{\frac{(\alpha+1) \varepsilon}{2}}$; and for $\varepsilon>0$ sufficiently small $\left(\varepsilon<\frac{2}{\alpha+1}\right)$, it holds that $C_{p}^{-\frac{(\alpha+1) \varepsilon}{2}} \stackrel{(c)}{\subseteq} C^{-\varepsilon} \stackrel{(d)}{\subseteq} C_{p}^{-\frac{(1+1 / \alpha) \varepsilon}{2}}$.

Before we prove Lemma 5.1, we address the following claim:

Claim 5.2 Let $C \subset \mathbb{R}^{d}$ be a compact convex set. Let $p$ be an $\alpha$-central point of $C$. Let $\ell$ be any line passing through $p$, and let $z$ and $y$ be the intersection points of $\ell$ with $\partial C$. Then for any two points $\beta_{1}$ and $\beta_{2}$ in $C$ such that $\beta_{1}-\beta_{2}$ is parallel to $p-z$, it holds that $\left|\beta_{1} \beta_{2}\right| \leqslant(1+\alpha)|p z|$.

Proof of Claim 5.2 Assume that $\left|\beta_{1} \beta_{2}\right| \geqslant 2|p z|$ (otherwise we are done) and that $\beta_{1}$ and $\beta_{2}$ are in $\partial C$. Consider the line passing through $\beta_{1}$ and $p$, and the line passing through $\beta_{2}$ and $z$. Denote their intersection by $w$ (the fact that $\left|\beta_{1} \beta_{2}\right| \geqslant 2|p z|$ assures that such an intersection exists). It is not hard to verify that $w \notin C^{o}$. We have that $\frac{\left|\beta_{1} \beta_{2}\right|}{|p z|}=\frac{\left|\beta_{1} w\right|}{|p w|} \leqslant \alpha+1$, where the last inequality follows from the fact that $p$ is central and from the properties of our construction. This suffices to prove our assertion.

Now we are ready to prove our lemma. 
Proof of Lemma 5.1 Notice that $C$ is included in $C_{p}^{\frac{(1+1 / \alpha) \varepsilon}{2}}, C^{\varepsilon}$, and $C_{p}^{\frac{(\alpha+1) \varepsilon}{2}}$. For (a), let $z$ be the intersection of $\partial C$ and the line passing through $p$ and $x \in C_{p}^{\frac{(1+1 / \alpha) \varepsilon}{2}} \backslash C$ (here $z$ is the intersection point closest to $x$ ). We have that $|x z| \leqslant \frac{(1+1 / \alpha) \varepsilon}{2}|p z|$ and that both $p$ and $z$ are in $C$, thus $x \in C \cup \partial_{\frac{\varepsilon}{2}} C=C^{\varepsilon}$. For (b), as before, let $z$ be the intersection of $\partial C$ and the line passing through $p$ and $x \in C^{\varepsilon} \backslash C=\left(C \cup \partial_{\frac{\varepsilon}{2}} C\right) \backslash C$. Let $c \in \partial C$, and $\beta_{1}$ and $\beta_{2}$ in $C$ such that $x=c+\frac{\varepsilon}{2}\left(\beta_{1}-\beta_{2}\right)$. Consider the intersection point $w$ between the line passing through $c$ and $z$ and the line passing through $p$ in the direction $\beta_{1}-\beta_{2}$. These lines intersect as all points $p, c, z$, and $x$ lie on a twodimensional plane. Both $c$ and $z$ are on $\partial C$, which implies that the point $w$ is not in $C^{o}$. Thus by Claim 5.2, it holds that $|p w| \geqslant\left|\beta_{1} \beta_{2}\right| /(1+\alpha)$. Now $\frac{|z x|}{|p z|}=\frac{|c x|}{|p w|} \leqslant$ $\frac{(1+\alpha) \varepsilon}{2}$, which implies that $x \in C_{p}^{\frac{(1+\alpha) \varepsilon}{2}}$.

For assertion (c), let $x \in C_{p}^{-(\alpha+1) \varepsilon / 2}$. Let $y, z$ be the intersection points of $\partial C$ and the line passing through $x$ and $p$ (where $z$ and $x$ are on the same side of $p$ ). It holds that $|x z| /|p z| \geqslant(\alpha+1) \varepsilon / 2$. We now show that $x+\frac{\varepsilon}{2}(C-C) \subseteq C$; this will suffice for our proof. For any vector $\beta_{1}-\beta_{2}$ in $C-C$, let $w$ be the intersection of the line passing through $p$ in direction $\beta_{1}-\beta_{2}$ with the boundary of $C$. By Claim 5.2 we have that $\left|\beta_{1} \beta_{2}\right| \leqslant(\alpha+1)|p w|$. Thus, $\frac{\varepsilon}{2}\left|\beta_{1} \beta_{2}\right| \leqslant \frac{\varepsilon(\alpha+1)}{2}|p w|$. This implies that $x+\frac{\varepsilon}{2}\left(\beta_{1}-\beta_{2}\right)$ is in the triangle $z w p$ (here we use the fact that $|x z| /|p z| \geqslant(\alpha+$ $1) \varepsilon / 2$ ), and thus in $C$. Finally, for (d), let $x \in C^{-\varepsilon}=C \backslash\left(\partial_{\frac{\varepsilon}{2}} C\right)^{o}$. Let $y$ and $z$ be the intersection of $\partial C$ and the line passing through $p$ and $x$ (as usual, $z$ is on the same side of $p$ as $x)$. It holds that $x \notin\left(z+\frac{\varepsilon}{2}(C-C)\right)^{o}$. Thus, the point $w=z+\frac{\varepsilon}{2}(y-z)$ is on the line segment $x z$. Namely, $|x z| \geqslant|w z|=\frac{\varepsilon}{2}|y z| \geqslant \frac{(1+1 / \alpha) \varepsilon}{2}|p z|$. We conclude that $x \in C_{p}^{-\frac{(1+1 / \alpha) \varepsilon}{2}}$.

We now show that the center of mass of $C$ and points of $C$ implied by John's theorem are $d$-central. We note that Theorems 1.1 and 1.2 can be proven (with slight modifications) when one considers the contraction $C_{p}^{-\varepsilon}$ for the points $p$ which are $\alpha$-central.

Claim 5.3 Let $C \subset \mathbb{R}^{d}$ be a compact convex set. Let $p$ be the center of mass of $C$, or alternatively let $p$ be a center point from John's theorem [13]. Let $\ell$ be any line passing through $p$, and let $z$ and $y$ be the intersection of $\ell$ with $\partial C$. It holds that $\frac{|z p|}{|y p|} \in\left[\frac{1}{d}, d\right]$.

Proof The proof is immediate for a point $p$ from John's theorem. We consider the center of mass $p$. Let $z$ and $y$ be the points that minimize the fraction $\frac{|z p|}{|y p|}$ (here we assume w.l.o.g. that $|z p| \leqslant|y p|)$, and let $\ell$ be the line connecting $y$ and $z$. Let $u$ be a vector such that $z$ is on the boundary of the slab $s_{u}(C)$. We will show that $\frac{|z p|}{|y p|} \geqslant \frac{1}{d}$. Let $p^{\prime}$ be the point on $\ell$ such that $\frac{\left|z p^{\prime}\right|}{\left|y p^{\prime}\right|}=\frac{1}{d}$. Let $H_{p^{\prime}}$ be the $u$-hyperplane passing through $p^{\prime}$, and let $C_{p^{\prime}}$ be the intersection of $C$ and $H_{p^{\prime}}$. Let $H_{z}$ be the $u$-hyperplane passing through $z$. Let $C_{z}$ be the set on $H_{z}$ obtained by a linear extension of $C_{p^{\prime}}$ with respect to $y$. Namely, for each point $\alpha_{p^{\prime}}$ in $C_{p^{\prime}}$, let $\alpha_{z}$ be the point (in $H_{z}$ ) obtained by the intersection of $H_{z}$ with the line passing through $y$ and $\alpha_{p^{\prime}}$. The set $C_{z}$ is defined 
to consist of all such points $\alpha_{z}$. Consider the minimum convex body $C^{*}$ consisting of the point $y$ and the set $C_{z}$. It is not hard to verify that the center of mass of $C^{*}$ lies on the hyperplane $H_{p^{\prime}}$. Moreover, $C$ includes the portion of $C^{*}$ that lies on the same side of $H_{p^{\prime}}$ as $y$ and is included in the portion of $C^{*}$ that lies on the same side of $H_{p^{\prime}}$ as $z$. We conclude that the center of mass $p$ of $C$ must lie in the line segment $p^{\prime} y$. This implies that $\frac{|z p|}{|y p|} \geqslant \frac{\left|z p^{\prime}\right|}{\left|y p^{\prime}\right|}=\frac{1}{d}$.

\subsection{Properties of Our Operations}

Lemma 5.2 (Continuity of contraction and expansion) Let $C$ be a compact convex set. For $\varepsilon>0,\left(C^{\varepsilon}\right)^{-\frac{\varepsilon}{1+\varepsilon}}=C$. If $\varepsilon>0$ is sufficiently small (namely $\varepsilon<\frac{2}{d+1}$ ), then $C \subseteq\left(C^{-\varepsilon}\right)^{\frac{(d+1) \varepsilon}{2-(d+1) \varepsilon}}$.

Proof For the first claim, let $\delta=\frac{\varepsilon}{1+\varepsilon}$. We first prove that $s_{u}\left(C^{\varepsilon}\right)=s_{u}^{\varepsilon}(C)$. Notice that $s_{u}(C)$ is included in both $s_{u}\left(C^{\varepsilon}\right)$ and $s_{u}^{\varepsilon}(C)$. For one direction, let $x \in s_{u}^{\varepsilon}(C) \backslash s_{u}(C)$. Let $y^{\prime}, z^{\prime}$ be the points on $\partial C$ that define $s_{u}(C)$ (take $z^{\prime}$ to be closer to $x$ than $y^{\prime}$ once all three are projected on $u$ ). Consider the line $\ell$ passing through $x$, in the direction $y^{\prime}-z^{\prime}$. Let $y, z$ be the intersection points of $\ell$ and the boundary of $s_{u}(C)$ (take $z$ to be closer to $x$ ). As $x \in s_{u}^{\varepsilon}(C) \backslash s_{u}(C)$, it holds that $|x z| /|x y|=\frac{\varepsilon^{\prime}}{2+\varepsilon^{\prime}}$ for some $\varepsilon^{\prime} \leqslant \varepsilon$. Let $x^{\prime}$ be on the line passing through $y^{\prime}$ and $z^{\prime}$ such that $\left|x^{\prime} z^{\prime}\right| /\left|x^{\prime} y^{\prime}\right|=\frac{\varepsilon^{\prime}}{2+\varepsilon^{\prime}}$. It holds that $x^{\prime} \in C^{(\varepsilon)}=C^{\varepsilon} \subseteq s_{u}\left(C^{\varepsilon}\right)$.

It remains to prove $s_{u}\left(C^{\varepsilon}\right) \subseteq s_{u}^{\varepsilon}(C)$. Assume that $x \in s_{u}\left(C^{\varepsilon}\right) \backslash s_{u}(C)$. Thus there exists $x^{\prime} \in C^{\varepsilon}$ such that $x-x^{\prime}$ is orthogonal to $u$. Namely, there are $y$ and $z$ in $C$ colinear with $x^{\prime}$ such that $\left|x^{\prime} z\right| /\left|x^{\prime} y\right| \leqslant \frac{\varepsilon}{2+\varepsilon}$. But then, if we consider the intersection of the line passing through $y$ and $z$ with $s_{u}(C)$, say at points $y^{\prime}$ and $z^{\prime}$ (with $y^{\prime}$ close to $y$ ), then $\left|z^{\prime} x^{\prime}\right| /\left|x^{\prime} y^{\prime}\right| \leqslant\left|x^{\prime} z\right| /\left|x^{\prime} y\right| \leqslant \frac{\varepsilon}{2+\varepsilon}$. This implies that $x^{\prime} \in s_{u}^{\varepsilon}(C)$. However, as $x-x^{\prime}$ is orthogonal to $u$, this also implies that $x \in s_{u}^{\varepsilon}(C)$. Now, $\left(C^{\varepsilon}\right)^{-\delta}=\bigcap_{u}\left(s_{u}\left(C^{\varepsilon}\right)\right)^{-\delta}=\bigcap_{u}\left(\left(s_{u}(C)\right)^{\varepsilon}\right)^{-\delta}=\bigcap_{u} s_{u}(C)=C$.

For the second claim, by John's theorem [13], there exists an ellipsoid $E$ centered at $p \in C$ such that $E \subseteq C \subseteq d(E-p)+p$. Assume w.l.o.g. that $p$ is the origin. Consider the contraction $C_{p}^{-\varepsilon}$ of $C$ with respect to $p$ (as defined in Sect. 5.2). Recall, by Lemma 5.1 and Claim 5.3, that $C_{p}^{-(d+1) \varepsilon / 2} \subseteq C^{-\varepsilon}$ (for $\varepsilon<\frac{2}{d+1}$ ). Now, as $A \subseteq B$ implies $A^{\varepsilon} \subseteq B^{\varepsilon}$ (for $\varepsilon>0$ ), we have $C=\frac{1}{1-(d+1) \varepsilon / 2}\left(C_{p}^{-(d+1) \varepsilon / 2}\right.$ ) $=$ $\left(C_{p}^{-(d+1) \varepsilon / 2}\right)^{\frac{(d+1) \varepsilon / 2}{1-(d+1) \varepsilon / 2}} \subseteq\left(C^{-\varepsilon}\right)^{\frac{(d+1) \varepsilon / 2}{1-(d+1) \varepsilon / 2}}$.

Remark 5.2 The bound on $\varepsilon$ in the second part of Lemma 5.2 may seem unnatural. However, it is necessary as for certain convex sets $C$, the contraction $C^{-\varepsilon}$ is empty once $\varepsilon$ is larger than $\frac{2}{d+1}$. Thus, any expansion of $C^{-\varepsilon}$ in this case remains empty. For example, consider the $d$-dimensional simplex $C=\left\{\left(x_{1}, \ldots, x_{d+1}\right) \in \mathbb{R}^{d+1}\right.$ $\left.\sum_{i=1}^{d+1} x_{i}=1\right\}$. For $x \in C$, it holds that $x$ has a coordinate of value at most $\frac{1}{d+1}$. It is not hard to verify that this implies that for $\varepsilon>\frac{2}{d+1}, C^{-\varepsilon}=\emptyset$. We note that if $\varepsilon \geqslant 2 /(d+1)$ and $C^{-\varepsilon}$ is not empty (and of full dimension), there exists an expansion parameter $\delta_{C}>0$ such that $C \subseteq\left(C^{-\varepsilon}\right)^{\delta_{C}}$. 
Using Theorem 5.1, we now obtain the following corollary of Lemma 5.2.

Corollary 5.1 (Continuity of contraction and expansion) For any sufficiently small $\varepsilon>0$, there exists $\delta>0$ such that for every positive $\varepsilon^{\prime}<\delta: C \subseteq\left(C^{\varepsilon}\right)^{-\varepsilon^{\prime}},\left(C^{\varepsilon^{\prime}}\right)^{-\varepsilon} \subseteq$ $C, C \subseteq\left(C^{-\varepsilon^{\prime}}\right)^{\varepsilon}$, and $\left(C^{-\varepsilon}\right)^{\varepsilon^{\prime}} \subseteq C$.

\section{Concluding Remarks}

In this work we have defined a notion of contraction and expansion of general convex sets and applied them in the proof of two Helly-type theorems. Our theorems are not constructive, as their proofs include points that may involve an exhaustive search over the set $\mathcal{S}$. The convex sets studied throughout were assumed to be compact (i.e., closed and bounded). For convex sets $C$ which are not closed, one may define the notion of contraction and expansion with respect to the closure of $C$. For convex sets $C$ which are unbounded, we extend the definition of one of the variants of $C^{\varepsilon}$ from Sect. 5 to the following:

Definition 6.1 Let $C$ be closed and convex, and let $\varepsilon$ be any real (positive, negative, or zero). If $|C| \leqslant 1$ or $C=\mathbb{R}^{d}$, let $C^{\varepsilon}=C$. Otherwise, let $r(x, C) \in[-1,1]$ be the closest point to 0 in the closure of the set

$$
\left\{\frac{\langle z-x, y-x\rangle}{\langle y-x, y-x\rangle}\right\}_{z \in \partial C, y \in C \quad x, y, z \text { colinear }} .
$$

Let $\|x\|_{C}=\frac{1+r(x, C)}{1-r(x, C)}$ (if $r(x, C)=1$, define $\|x\|_{C}$ to be $\infty$ ). Let $C^{\varepsilon}=\left\{x \mid\|x\|_{C} \leqslant\right.$ $1+\varepsilon\}$.

The above definition differs from that presented in Sect. 5 in the restriction that $y \in C$ instead of $y \in \partial C$. In general, for convex sets $C$ with the property that any infinite line in $\mathbb{R}^{d}$ either intersects $\partial C$ in two points, is tangent to $C$, or does not intersect $\partial C$ at all, the definition above and that of Sect. 5 are equivalent. We refer to such sets as proper. For example, bounded convex sets are proper. Sets $C$ that are not proper are sets which are in essence similar to half spaces-roughly speaking, they consist of the (Minkowski) sum $C^{\prime}+\ell$ for some convex set $C^{\prime}$ and an infinite (one-dimensional) ray $\ell$; or alternatively, they are contained in a half-space $H$ and contain points arbitrarily far from $\partial H$.

The proof of Theorem 1.1 can be altered to deal with sets $C$ in $\mathcal{S}$ that may be unbounded and proper. Namely, one needs to consider an unbounded and proper set $A=\bigcap_{C \in \mathcal{S}} C$. Such $A$ includes an infinite line $\ell$, which is also included in $C$ for each $C \in \mathcal{S}$. As the sets considered are all proper, this implies that $C=\ell+C^{\prime}$ where $C^{\prime}$ is a $(d-1)$-dimensional convex set in the hyperplane orthogonal to $\ell$ passing through the origin. We may thus reduce the dimension of the problem by considering the collection $\mathcal{S}=\left\{C^{\prime}\right\}_{C \in \mathcal{S}}$.

It is now simple to verify that our theorems hold for all convex sets $C$ (proper or not). This follows by the fact that $C^{\varepsilon}=\emptyset$ for any $\varepsilon<0$ when $C$ is not proper. 
Specifically, Theorem 1.1 holds trivially when $\mathcal{S}$ contains a set which is not proper, and in Theorem 1.2 one can discard of sets that are not proper and only consider the sets in each $C_{i}$ that are proper (for the latter, unbounded sets $C$ are not considered to be $f$-fat for any value of $f$ ).

Acknowledgements We would like to thank Jie Gao for several helpful discussions which led to the study of contraction and expansion of convex sets. We would also like to thank Gil Kalai, Micha Perles, and Jiri Matoušek for helpful discussions about this work. Finally, we would like to thank Micha Sharir for pointing out the work of Dudley [6].

\section{References}

1. Alon, N., Kalai, G.: Bounding the piercing number. Discrete Comput. Geom. 13(3/4), 245-256 (1995)

2. Amenta, N.: A short proof of an interesting Helly-type theorem. Discrete Comput. Geom. 15(4), 423427 (1996)

3. Bárány, I., Pach, M.K.J.: Quantitative Helly-type theorems. Proc. Am. Math. Soc. 86(1), 109-114 (1982)

4. Barvinok, A.: A Course in Convexity. Graduate Studies in Mathematics, vol. 54. Am. Math. Soc., Providence (2002)

5. Demouth, J., Devillers, O., Glisse, M., Goaoc, X.: Helly-type theorems for approximate covering. In: Proceedings of the Twenty-Fourth Annual Symposium on Computational Geometry, pp. 120-128 (2008)

6. Dudley, R.M.: Metric entropy of some classes of sets with differentiable boundaries. J. Approx. Theory 10(3), 227-236 (1974)

7. Eckhoff, J.: Helly, Radon, and Carathéodory type theorems. In: Gruber, P.M., Wills, J.M. (eds.) Handbook of Convex Geometry, vol. A, pp. 389-448. North-Holland, Amsterdam (1993)

8. Efrat, A., Katz, M.J., Nielsen, F., Sharir, M.: Dynamic data structures for fat objects and their applications. Comput. Geom. 15(4), 215-227 (2000)

9. Gao, J., Langberg, M., Schulman, L.J.: Clustering lines: classification of incomplete data. Manuscript (2006)

10. Gruber, P.M.: Aspects of approximation of convex bodies. In: Handbook of Convex Geometry, vol. A, pp. 319-345. North-Holland, Amsterdam (1993)

11. Grunbaum, B., Motzkin, T.S.: On components in some families of sets. Proc. Am. Math. Soc. 12(4), 607-613 (1961)

12. Helly, E.: Über Mengen konvexer Körper mit gemeinschaftlichen Punkten. Jahresber. Dtsch. Math.Ver. 32, 175-176 (1923)

13. John, F.: Extremum problems with inequalities as subsidiary conditions. In: Studies and Essays Presented to R. Courant on his 60th Birthday, pp. 187-204. Interscience, New York (1948)

14. Kalai, G., Meshulam, R.: Leray numbers of projections and a topological Helly-type theorem. J. Topol. 1(3), 551-556 (2008)

15. Larman, D.G.: Helly type properties of unions of convex sets. Mathematika 15, 53-59 (1968)

16. Matoušek, J.: A Helly-type theorem for unions of convex sets. Comput. Geom. 18(1), 1-12 (1997)

17. Morris, H.: Two pigeonhole principles and unions of convexly disjoint sets. Ph.D. Thesis, Calif. Inst. of Techn., Calif. (1973)

18. Rademacher, H., Schoenberg, I.J.: Helly's theorem on convex domains and Tchebycheff's approximation problem. Can. J. Math. 2, 245-256 (1950)

19. Shnirelman, L.G.: On uniform approximations. Izv. Akad. Nauk SSSR Ser. Mat. 2, 53-60 (1938)

20. van der Stappen, A.F., Halperin, D., Overmars, M.H.: The complexity of the free space for a robot moving amidst fat obstacles. Comput. Geom. Theory Appl. 3, 353-373 (1993)

21. Wenger, R.: Helly-type theorems and geometric transversals. In: Handbook of Discrete and Computational Geometry, pp. 63-82. CRC Press, Boca Raton (1997) 